\title{
$\mathbb{B} \Delta+$ COMMUNICATIONS MATERIALS
}

ARTICLE

OPEN

https://doi.org/10.1038/s43246-020-00075-1

\section{Making EuO multiferroic by epitaxial strain engineering}

\author{
Veronica Goian (1) ${ }^{1}$, Rainer Held ${ }^{2}$, Eric Bousquet (10 ${ }^{3}$, Yakun Yuan ${ }^{4,5}$, Alexander Melville ${ }^{2}$, Hua Zhou (1) ${ }^{6}$, \\ Venkatraman Gopalan (1D 4,5,7, Phillipe Ghosez ${ }^{3}$, Nicola A. Spaldin (1) ${ }^{8}$, Darrell G. Schlom (1D ${ }^{2,9,10}$ \& \\ Stanislav Kamba (1D ${ }^{1 凶}$
}

Multiferroics are materials exhibiting the coexistence of ferroelectricity and ideally ferromagnetism. Unfortunately, most known magnetoelectric multiferroics combine ferroelectricity with antiferromagnetism or with weak ferromagnetism. Here, following previous theoretical predictions, we provide clear experimental indications that ferroelectricity can be induced by epitaxial tensile strain in the ferromagnetic simple binary oxide EuO. We investigate the ferroelectric phase transition using infrared reflectance spectroscopy, finding that the frequency of the soft optical phonon reduces with increasing tensile strain and decreasing temperature. We observe such a soft mode anomaly at $100 \mathrm{~K}$ in $(\mathrm{EuO})_{2} /(\mathrm{BaO})_{2}$ superlattices grown epitaxially on $\left(\mathrm{LaAlO}_{3}\right)_{0.29}-\left(\mathrm{SrAl}_{1 / 2} \mathrm{Ta}_{1 / 2} \mathrm{O}_{3}\right)_{0.71}$ substrates, which is a typical signature for a displacive ferroelectric phase transition. The EuO in this superlattice is nominally subjected to $6.4 \%$ biaxial tensile strain, i.e., $50 \%$ more than believed needed from previously published calculations. We interpret our results with new first-principles density functional calculations using a hybrid functional, which provides a better quantitative agreement with experiment than the previously used local-density approximation and generalized gradient approximation functionals.

\footnotetext{
${ }^{1}$ Institute of Physics of the Czech Academy of Sciences, Na Slovance 2, 18221 Prague 8, Czech Republic. ${ }^{2}$ Department of Materials Science and Engineering, Cornell University, Ithaca, NY 14853, USA. ${ }^{3}$ Physique Théorique des Matériaux, Q-MAT, CESAM, Université de Liège, B-4000 Sart Tilman, Belgium.

${ }^{4}$ Department of Materials Science and Engineering, Pennsylvania State University, University Park, PA 16802, USA. ${ }^{5}$ Materials Research Institute, Pennsylvania State University, University Park, PA 16802, USA. ${ }^{6}$ Advanced Photon Source, Argonne National Laboratory, Lemont, IL 60439, USA.

7 Department of Physics, Pennsylvania State University, University Park, PA 16802, USA. ${ }^{8}$ Materials Theory, ETH Zurich, CH-8093 Zürich, Switzerland.

${ }^{9}$ Kavli Institute at Cornell for Nanoscale Science, Ithaca, NY 14853, USA. ${ }^{10}$ Leibniz-Institut für Kristallzüchtung, Max-Born-Str. 2,12489 Berlin, Germany.

凶email: kamba@fzu.cz
} 
E pitaxial strain has proved to be an effective thin film control parameter to enhance properties or to induce new phases with functionalities absent in the unstrained parent bulk compound. For example, it can increase the superconducting, ferroelectric, or ferromagnetic transition temperatures, and improve microwave dielectric properties; it can even induce ferroelectric or ferromagnetic states in materials where these features are not present in the absence of strain ${ }^{1-4}$. This approach has been applied to enlarge the limited number of multiferroics ${ }^{5-9}$, which are simultaneously ferroelectric and (anti)ferromagnetic, with enhancement of their magnetoelectric response ${ }^{10-13}$.

Several such enhancements were first predicted by theory and simulations, mostly density functional theory (DFT), before their experimental confirmation. One of these examples is the perovskite $\mathrm{EuTiO}_{3}$, an antiferromagnetic incipient ferroelectric in which $1 \%$ tensile strain induces ferroelectricity and ferromagnetism, making it multiferroic ${ }^{5,14}$. Other recent successful examples are the cases of $\mathrm{CaMnO}_{3}{ }^{15}$ in which incipient ferroelectricity was observed below $25 \mathrm{~K}$ under $2.4 \%$ tensile strain ${ }^{16}$, or $\mathrm{SrMnO}_{3}{ }^{6}$, in which a phase transition to a polar state at $380 \mathrm{~K}$ has been observed for $1.7 \%$ tensile strain ${ }^{17}$, while the antiferromagnetic transition temperature is simultaneously shifted from the bulk value of $230 \mathrm{~K}^{18,19}$ to $180 \mathrm{~K}$ in the strained film ${ }^{19}$. Another interesting case is strained $\mathrm{NaMnF}_{3}$, which was predicted to be ferroelectric with a weak ferromagnetic moment from DFT simulations ${ }^{8}$ and the multiferroic behavior was subsequently confirmed experimentally in films grown on $\mathrm{SrTiO}_{3}$ substrates 9 .

Beyond the perovskite family, strain-induced ferroelectricity has also been predicted for the simple binary oxides $\mathrm{SrO}, \mathrm{BaO}$, $\mathrm{EuO}^{7}$ as well as in other binary compounds with the rocksalt structure: II-VI sulfides, selenides, and I-VII halides ${ }^{20}$. In all cases, however, the required strain was rather high and no experiment has previously confirmed these predictions. The case of EuO is particularly attractive due to its excellent potential for spintronic applications: it possesses strong magneto-optic activity 21,22 , colossal magnetoresistance ${ }^{23,24}$, anomalous Hall effect $^{25}$, high spin polarization ${ }^{26}$, and it can be epitaxially integrated with silicon ${ }^{27}$. Adding ferroelectric and multiferroic properties to these alluring features would make EuO even more appealing for technological applications. Additionally, inducing ferroelectricity in $\mathrm{EuO}$ while maintaining its ferromagnetism (it has a large magnetic moment of $7 \mu_{\mathrm{B}}$ /atom and a modest ferromagnetic Curie temperature of $69 \mathrm{~K}$ ) would make it a strong multiferroic (i.e., one that combines ferroelectricity with pure ferromagnetism and not with antiferromagnetism), an extremely rare property in crystals.

In the present paper, we show that $\mathrm{EuO}$ films and $\mathrm{EuO} / \mathrm{BaO}$ superlattices grown by molecular-beam epitaxy (MBE) on different substrates of increasing epitaxial strain (from $+2.2 \%$ to $+6.4 \%$ ) exhibit softening and condensation of a polar phonon in the EuO layers associated with a divergence of the dielectric constant; this is the hallmark of a ferroelectric phase transition. We also show that the $\mathrm{EuO}$ layers retain their ferromagnetic character under these conditions, which thus confirms the sought-after strain-induced multiferroicity. To better understand the experimental results, we report new and more accurate DFT studies of strain-induced ferroelectricity in EuO. These calculations show that the predicted critical biaxial tensile strain to destabilize the polar order is higher $(+5.8 \%)$ than the previously reported prediction $(+4 \%)^{7}$, and more consistent with our experimental observations.

\section{Results and discussion}

Infrared studies of relaxed and tensile-strained $\mathrm{EuO}$ films. To measure the high-frequency dielectric properties and optical phonons, infrared (IR) reflectance spectroscopy was used. This technique is highly sensitive. It has been demonstrated on ultrathin films with thicknesses as small as $20 \mathrm{~nm}$ in several materials-see e.g., the recent review of Petzelt and Kamba ${ }^{28}$. In addition, IR spectra are not influenced by leakage conductivity, which frequently precludes the direct low-frequency dielectric study of ultrathin films. Using this technique, we show that biaxial strain indeed noticeably reduces the phonon frequency in $\mathrm{EuO}$, with a critical biaxial strain for ferroelectricity of around $+6 \%$.

Biaxially straining EuO more than $5 \%$ is challenging, as such films tend to relax after the epitaxial growth of only a few monolayers. We overcame this challenge by growing superlattices made of $\mathrm{EuO}$ and $\mathrm{BaO}$ at growth temperatures at or below $125^{\circ} \mathrm{C}$, with a periodicity that contains only a few monolayers of each. When deposited under the conditions described in the Supplementary Information, these structures permit high strain levels to be achieved in films thick enough to investigate using IR reflectance. The strain to the EuO layers in the superlattice is imposed by the lattice parameter of the substrate and verified by X-ray diffraction (see Supplementary Figs. 1-4). Provided a substrate with a lattice parameter larger than that of $\mathrm{EuO}$ $\left(a_{\mathrm{EuO}}=5.145 \AA\right)$ and smaller than that of $\mathrm{BaO}\left(a_{\mathrm{BaO}}=5.539 \AA\right)$ is employed, the number of monolayers $x$ and $y$ of the $(\mathrm{EuO})_{x} /$ $(\mathrm{BaO})_{y}$ superlattice can be chosen to make the superlattice as a whole well matched to the underlying substrate, even though significant strains are imposed on the $\mathrm{EuO}$ and $\mathrm{BaO}$ constituent layers of the superlattice. Since the superlattice as a whole is nearly matched to the substrate, the superlattice can be repeated many times to increase the total volume of strained $\mathrm{EuO}$ in the structure without relaxing the strain in the $\mathrm{EuO}$ layers of the superlattice. In this way, we were able to grow highly strained films with sufficient thickness to investigate by IR reflectance.

We first measured the optical phonon in unstrained epitaxial (001) EuO films grown on (001) yttria-stabilized cubic zirconia (YSZ), $\left(\mathrm{Y}_{2} \mathrm{O}_{3}\right)_{0.095}\left(\mathrm{ZrO}_{2}\right)_{0.905}$, substrates. This substrate is almost exactly lattice-matched with $\mathrm{EuO}$ (the difference in lattice constant is only $0.04 \%$ ). The bare YSZ substrate exhibits broad reflection bands (Fig. 1), which are almost independent of temperature. The sharp peak seen in Fig. 1 near $180 \mathrm{~cm}^{-1}$ corresponds to the EuO transverse optical phonon. Note that the frequency is slightly lower than the $199 \mathrm{~cm}^{-1}$ published by Axe, who measured EuO single crystals ${ }^{29}$. Since EuO crystallizes in the cubic rocksalt crystal structure, only one triply degenerate IR-active phonon is allowed in the spectrum. Damping of the phonon strongly decreases on cooling; therefore, the $\mathrm{EuO}$

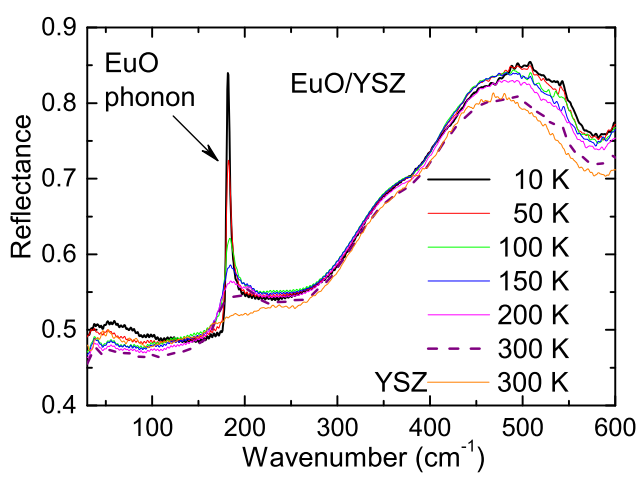

Fig. 1 Temperature dependence of the IR reflectance spectra of an EuO film grown on a (001) YSZ substrate. The $40 \mathrm{~nm}$ thick EuO film is protected by a $20 \mathrm{~nm}$ a-Si cap. The spectrum at room temperature of a YSZ substrate (thickness $1 \mathrm{~mm}$ ) capped with a-Si is also shown. The spectrum of bare YSZ has the same shape (not shown). 


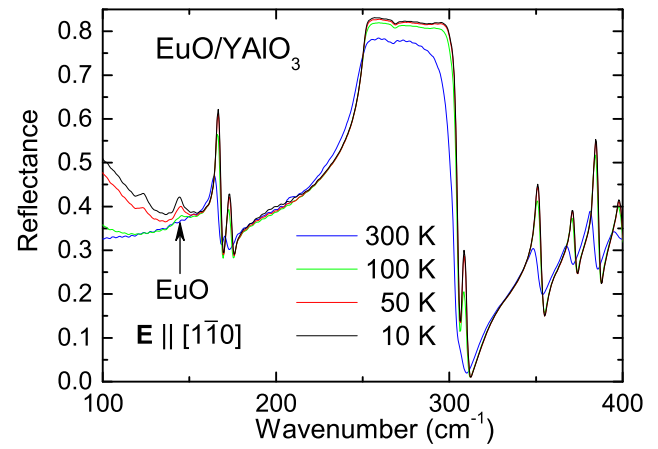

Fig. 2 Temperature dependence of the IR reflectance of a $15 \mathrm{~nm}$ thick EuO film (strain $+2.2 \%$ ) grown on a (110) $\mathrm{YAIO}_{3}$ substrate. The spectra were measured with polarization $\mathbf{E}||[110]$. Most of the reflection bands correspond to substrate phonons. The only EuO phonon is marked by the arrow. The increase of the reflectance below $140 \mathrm{~cm}^{-1}$ is due to reflection of the beam from the back side of the substrate, which is covered by a Pt layer used to couple to the radiative heater during film deposition. This increase is seen only at low temperatures because the substrate is transparent in this frequency range only at low temperatures.

reflectance peak sharpens and its intensity increases as the temperature is lowered. Its frequency slightly hardens from 180 $\mathrm{cm}^{-1}$ at room temperature to $182 \mathrm{~cm}^{-1}$ at $10 \mathrm{~K}$ due to thermal contraction on cooling.

The second set of EuO films was grown on (110) $\mathrm{YAlO}_{3}$. Since this substrate is orthorhombic $(a=5.180 \AA, b=5.330 \AA, c=$ $7.375 \AA)^{30}$, it nominally induces anisotropic tensile strains of $+1.5 \%$ along the [001] and $+2.2 \%$ along the [1ㅣㅣ directions, respectively. In Fig. 2 we show only the experimental spectra with polarization $\mathrm{E} \|[1 \overline{1} 0]$ (i.e., along the highest strain direction). Our fit of the spectra revealed the phonon frequency to be 150 $\mathrm{cm}^{-1}$ (at room temperature) and softening to $144 \mathrm{~cm}^{-1}$ on cooling to $10 \mathrm{~K}$. Studies of the phonon density of states (PDOS) in a similar (001) EuO film on (110) $\mathrm{YAlO}_{3}$ using nuclear inelastic scattering revealed also only small shift of PDOS to lower energies $^{31}$.

Lattice dynamics calculations in strained $\mathrm{EuO}$ and $\mathrm{BaO}$. The observed softening of the optical phonon of $\mathrm{EuO}$ for unstrained $\left(182 \mathrm{~cm}^{-1}\right.$ at $\left.10 \mathrm{~K}\right)$ vs. $+2.2 \%$ strain $\left(144 \mathrm{~cm}^{-1}\right.$ at $\left.10 \mathrm{~K}\right)$ from experiment, a change of $21 \%$, is far less than the $\sim 40 \%$ softening expected from previously published calculations ${ }^{7}$. This lower than expected softening suggests that the critical strain for ferroelectricity in biaxially strained (001) $\mathrm{EuO}$ is higher than the $+4 \%$ predicted $^{7}$. To better understand this discrepancy between our observations and calculations made using both the local-density approximation (LDA) as well as the generalized gradient approximations $(\mathrm{GGA})^{7}$, we performed new calculations of the dependence of the soft mode in EuO using the hybrid-functional $\mathrm{B}_{1} \mathrm{WC}^{32}$ as implemented in the CRYSTAL code ${ }^{33}$. Hybrid functionals have been shown to give better phonon frequencies in bulk $\mathrm{BaO}^{20}$ and the $\mathrm{B} 1 \mathrm{WC}$ functional in particular describes the structural and electronic properties of ferroelectric perovskites well ${ }^{34}$.

Consistent with the earlier calculations for $\mathrm{BaO}$ and our expectation for $\mathrm{EuO}$, we found that the calculated $\mathrm{B} 1 \mathrm{WC}$ transverse optic frequencies of bulk $\mathrm{BaO}$ and $\mathrm{EuO}$ are in better agreement with the experiment than the previously reported LDA and GGA values. We obtained transverse optic phonon frequencies of $136 \mathrm{~cm}^{-1}$ for bulk $\mathrm{BaO}$ (vs. $145 \mathrm{~cm}^{-1}$ from experiment ${ }^{35,36}$ and $101 \mathrm{~cm}^{-1}$ with $\mathrm{LDA}^{7}$ or $96 \mathrm{~cm}^{-1}$ with GGA-WC ${ }^{7}$ ) and $203 \mathrm{~cm}^{-1}$ for bulk EuO (vs. $199 \mathrm{~cm}^{-1}$ from experiment ${ }^{29}$ and $164 \mathrm{~cm}^{-1}$ in $\mathrm{GGA}^{\mathrm{PBE}^{7}}$ ). We then computed

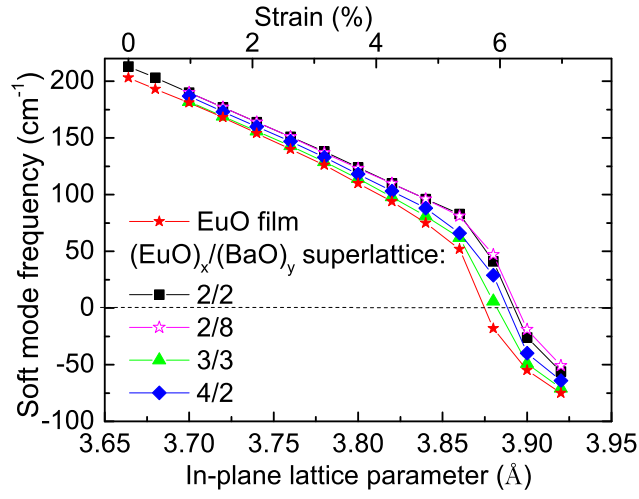

Fig. 3 Theoretical strain dependence of the EuO phonon frequency in a biaxially strained (001) EuO film and in various $(\mathrm{EuO})_{x} /(\mathrm{BaO})_{y}$ superlattices. The B1WC hybrid functional was used for the calculations. Negative numbers on the vertical axis correspond to imaginary frequencies and a lattice instability.

the evolution of the EuO transverse phonon frequency versus strain with the B1WC functional and found a critical strain of about $+5.8 \%$ is needed to destabilize the polar mode (see Fig. 3, red curve). This means that the $\mathrm{B} 1 \mathrm{WC}$ functional predicts a larger critical strain for ferroelectricity in $\mathrm{EuO}$ than that obtained using $\mathrm{GGA}^{7}$ and thus is more consistent with our experimental results. Although we tried, we were unable to grow EuO films of sufficient thickness to measure the phonon by IR reflectivity at this high strain value.

Since we were not able to attain such high strain levels with conventional coherent heteroepitaxy, we shifted the strategy of our experiment and extended our hybrid functional DFT calculations to the case of strained $(\mathrm{EuO})_{x} /(\mathrm{BaO})_{y}$ superlattices. As the lattice parameter of $\mathrm{EuO}$ is smaller than that of $\mathrm{BaO}$, substrates with lattice spacings between $\mathrm{EuO}$ and $\mathrm{BaO}$ will induce tensile strain in $\mathrm{EuO}$ and compressive strain in $\mathrm{BaO}$. The number of monolayers $x$ and $y$ in these $(\mathrm{EuO})_{x} /(\mathrm{BaO})_{y}$ superlattices are chosen to make the superlattice as a whole well matched to the underlying substrate. This enables the superlattice to be repeated many times to increase the total volume of strained $\mathrm{EuO}$ in the structure without relaxing the strain in the EuO layers of the superlattice. This "strained-layer superlattice" strategy is commonly used in the growth of semiconductors ${ }^{37}$.

In Fig. 3 we report the theoretical evolution of the soft mode frequency as a function of in-plane lattice parameter for a range of $x / y$ periodicities; the upper horizontal axis gives the corresponding tensile strain in the $\mathrm{EuO}$ layer. Imaginary frequencies, indicating unstable modes, are plotted on the negative $y$-axis. We see that all of the periodicities have a critical strain for destabilization of the polar mode. The 2/2, 2/6 (not shown), and $2 / 8$ combinations have critical strains of about $6.2 \%$, which is larger than that of the homogeneous film; the $3 / 3$ and $4 / 2$ heterostructures have lower critical strain than $2 / y$ superlattices, but slightly higher than pure EuO. The reason for this effect can be attributed to the fact that $\mathrm{BaO}$ is under compressive strain, which does not favor in-plane ferroelectricity. This, in turn, disfavors in-plane polar displacements in the $\mathrm{EuO}$ at the interface with $\mathrm{BaO}$. This is confirmed by the fact that for larger thickness of EuO the critical strain is closer to that of pure EuO. We notice that in the strained region where the $E_{u}$ symmetry mode (polarized in the film plane-see the schematic shown in the inset of Fig. 4) is destabilized in $\mathrm{EuO}, \mathrm{BaO}$ is under insufficient compressive strain to induce ferroelectricity in $\mathrm{BaO}$. The $A_{2 u}$ component of the $\mathrm{BaO}$ phonon, which is polarized outof-plane, can be softer than in bulk $\mathrm{BaO}$. This $A_{2 u}$ component 

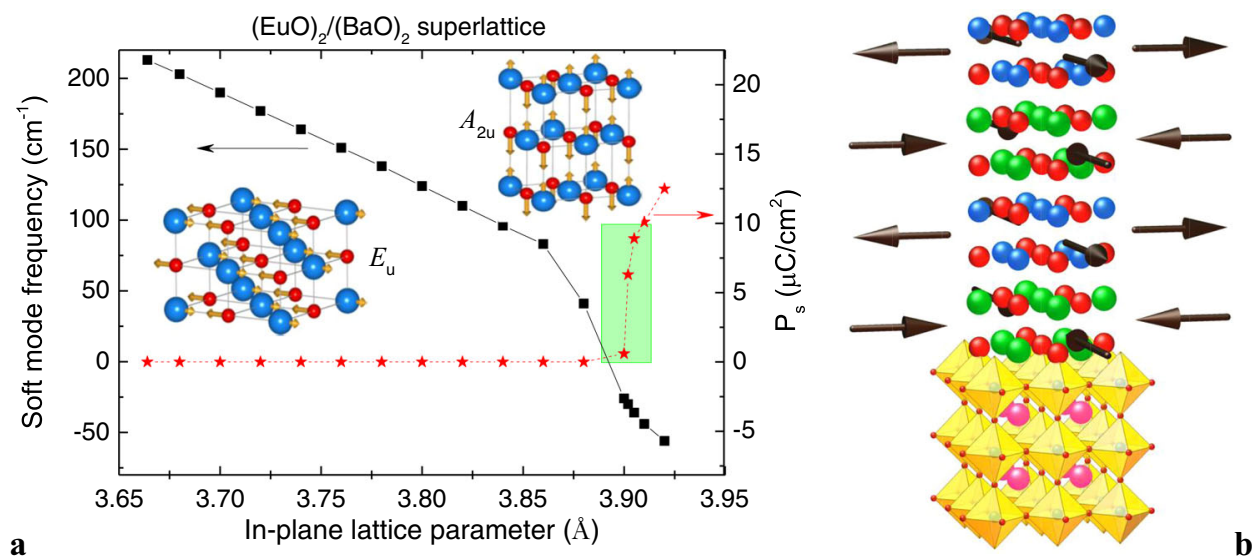

Fig. 4 Schematic illustration of an $(\mathrm{EuO})_{2} /(\mathrm{BaO})_{2}$ superlattice on a (001) LSAT substrate and the theoretical strain dependence of the soft mode frequency and the spontaneous polarization in the EuO layers of the superlattice. a The strain dependence of the frequency of the $E_{\mathrm{u}}$ ferroelectric soft mode (left scale) and the spontaneous polarization $P_{\mathrm{s}}$ (right scale) in an $(\mathrm{EuO})_{2} /(\mathrm{BaO})_{2}$ superlattice obtained from the DFT calculations. The green bar reflects the range of DFT accuracy for the EuO strain. Insets show schematic representations of the $E_{\mathrm{u}}$ and $A_{2 \mathrm{u}}$ symmetry polar vibrations. $\mathbf{b}$ Crystal structure of the $(\mathrm{EuO})_{2} /(\mathrm{BaO})_{2}$ superlattice on an (001) LSAT perovskite substrate in which the $(\mathrm{EuO})_{2}$ layer is under biaxial tension and the $(\mathrm{BaO})_{2}$ layer is under biaxial compression. The directions of both strains are marked by arrows. Eu, $\mathrm{Ba}$, and $\mathrm{O}$ atoms are shown in blue, green, and red, respectively.

cannot, however, couple with the $E_{u}$ soft mode in $\mathrm{EuO}$, because they have different symmetries, i.e., electrostatic coupling between layers in a superlattice is not allowed.

In Fig. 4 we report the calculated evolution of both the $E_{u}$ soft mode frequency of the high-symmetry paraelectric phase (space group P4/nmm number 129) and the amplitude of the spontaneous polarization $P_{\mathrm{s}}$ of the low symmetry ferroelectric phase (space group $P m n 2_{1}$ number 31) of the $(\mathrm{EuO})_{2} /(\mathrm{BaO})_{2}$ superlattice with respect to the imposed in-plane unit cell parameter. We can see that the critical lattice parameter for the destabilization of the polar mode and the associated appearance of non-zero polarization is between 3.89 and $3.90 \AA$. The primitive unit cell parameter of unstrained bulk EuO is $3.664 \AA$, making the predicted critical strain between $6.18 \%$ and $6.45 \%$. The accuracy of the B1WC functional in predicting the unit cell parameter of $\mathrm{EuO}$ is about $0.7 \%$. This uncertainly leads to the predicted polarization of the commensurately strained superlattice on LSAT being between 0.6 and $10 \mu \mathrm{C} / \mathrm{cm}^{2}$ (marked in Fig. 4).

Infrared studies of strained $(\mathrm{EuO})_{x} /(\mathrm{BaO})_{y}$ superlattices. Motivated by these calculations supporting a change in experimental strategy from a single layer of $\mathrm{EuO}$ to $\mathrm{EuO}$ within a strained-layer superlattice, we prepared $(\mathrm{EuO})_{2} /(\mathrm{BaO})_{2}$ superlattices with 35 repetitions, i.e., $\left[(\mathrm{EuO})_{2} /(\mathrm{BaO})_{2}\right]_{35}$, on $(001)$ $\left(\mathrm{LaAlO}_{3}\right)_{0.29^{-}}\left(\mathrm{SrAl}_{1 / 2} \mathrm{Ta}_{1 / 2} \mathrm{O}_{3}\right)_{0.71}$ (abbreviated as LSAT) substrates. To protect the superlattices from degradation they were capped with $\sim 100 \mathrm{~nm}$ of amorphous silicon (a-Si). In this case, the $\mathrm{EuO}$ and $\mathrm{BaO}$ are nominally strained by $+6.4 \%$ and $-1.2 \%$, respectively. The strains in these superlattices are high and even with accurate calibration of deposition rates and a low growth temperature of $\sim 80^{\circ} \mathrm{C}$ it was challenging to maintain the strain during growth. The superlattice with the highest structural perfection was selected for IR reflectance measurements; the rocking curve full width at half maximum of this best sample was $0.13^{\circ}$ (see Supplementary Fig. 2).

In Fig. 5 we show the far-IR reflectance spectra of the $\left[(\mathrm{EuO})_{2} /\right.$ $\left.(\mathrm{BaO})_{2}\right]_{35} /$ LSAT sample. At first sight, no dramatic changes with temperature are observed in the reflectance: the phonon reflection band seen close to $170 \mathrm{~cm}^{-1}$ belongs to the LSAT substrate ${ }^{38}$ and shows only classical sharpening upon cooling. Nonetheless, careful analysis reveals an increase of the reflectance in the

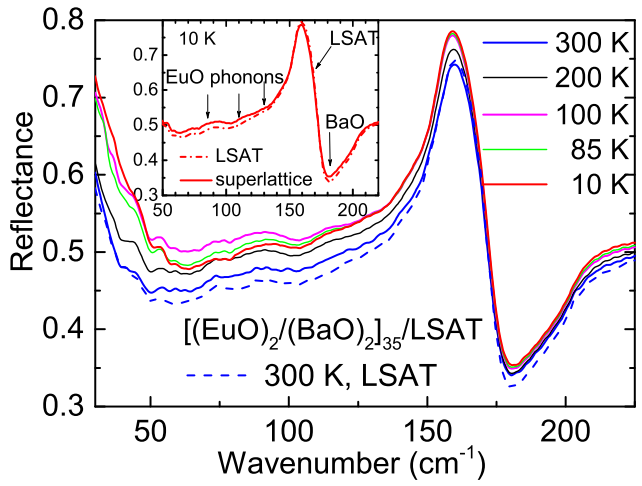

Fig. 5 Temperature dependence of the far-IR reflectance of an [(EuO $)_{2} /$ $\left.(\mathrm{BaO})_{2}\right]_{35}$ superlattice. The superlattice with thickness $35.8 \mathrm{~nm}$ was grown on a (001) LSAT substrate. Spectra from a bare (001) LSAT substrate are shown with dashed lines. The increase of the reflectance below $50 \mathrm{~cm}^{-1}$ is due to the reflection of the beam from the backside of the semitransparent substrate in this frequency range. The inset shows spectra of a bare LSAT substrate and the superlattice at $10 \mathrm{~K}$.

$50-100 \mathrm{~cm}^{-1}$ range on cooling towards $100 \mathrm{~K}$ followed by a decrease on further cooling down to $10 \mathrm{~K}$. This suggests a phonon softening and subsequent hardening in this frequency region, which is typical for a ferroelectric phase transition.

To confirm this finding, in Fig. 6 we report the complex dielectric spectra of $\mathrm{EuO}$ and $\mathrm{BaO}$ layers obtained from careful fits of the $\left[(\mathrm{EuO})_{2} /(\mathrm{BaO})_{2}\right]_{35} / \mathrm{LSAT}$ superlattice data. One can clearly see noticeable softening of the EuO phonon towards $100 \mathrm{~K}$ and again it is hardening below this temperature. This is also confirmed from the EuO static relative permittivity $\varepsilon_{0}^{\prime}$ (calculated from Eq. 3) plotted in Fig. 7, which increases from 280 at $300 \mathrm{~K}$ to 600 at $100 \mathrm{~K}$ and again decreases below $100 \mathrm{~K}$ in the case of the $\left[(\mathrm{EuO})_{2} /(\mathrm{BaO})_{2}\right]_{35} /$ LSAT superlattice (red curve on Fig. 7$)$, while the other strain cases do not show this anomaly (blue, black, and green curves). The phonon and dielectric anomaly observed at $100 \mathrm{~K}$ is characteristic of a displacive ferroelectric phase transition. This conclusion is strengthened by the appearance of new phonon modes below $100 \mathrm{~K}$ with frequencies of 110 and 130 $\mathrm{cm}^{-1}$ (see Fig. 8, red curves), which is the signature of symmetry lowering below $100 \mathrm{~K}$ expected in the ferroelectric phase. Here it 


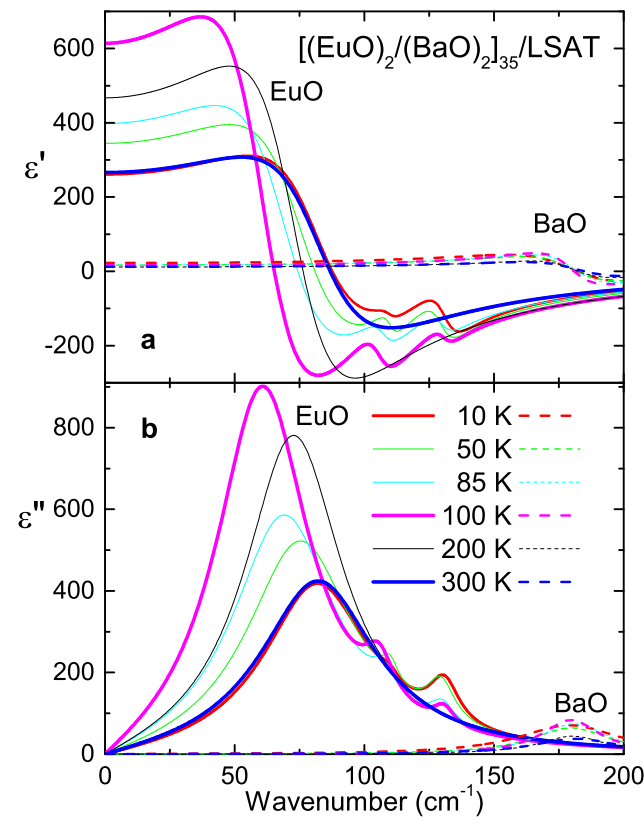

Fig. 6 Complex dielectric response of EuO and $\mathrm{BaO}$ layers in an $\left[(\mathrm{EuO})_{2} /\right.$ $\left.(\mathrm{BaO})_{2}\right]_{35}$ superlattice grown on a (001) LSAT substrate. (a) Real and (b) imaginary parts of the complex dielectric function in $\mathrm{EuO}$ (solid lines, biaxial tensile strain $+6.4 \%$ ) and $\mathrm{BaO}$ (dashed lines, compressive strain $-1.2 \%$ ) layers were obtained from fits to the IR spectra of the $\left[(\mathrm{EuO})_{2} /(\mathrm{BaO})_{2}\right]_{35} /$ LSAT sample in Fig. 5. A higher phonon frequency and lower relative permittivity are seen in the $\mathrm{BaO}$ layers due to the compressive strain of the $\mathrm{BaO}$. A strong ferroelectric soft mode with the lowest frequency at $100 \mathrm{~K}$ is clearly seen in the EuO.

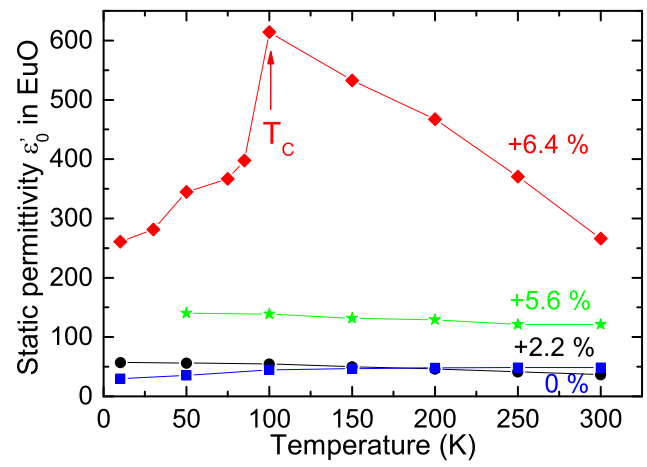

Fig. 7 Temperature and strain dependence of the static relative permittivity of the EuO films and EuO layers in the (EuO) $)_{x} /(\mathrm{BaO})_{y}$ superlattices. The permittivity values were calculated from fits of the IR reflectance. The ferroelectric critical temperature observed for the $\left[(\mathrm{EuO})_{2} /(\mathrm{BaO})_{2}\right]_{35}$ superlattice strained to LSAT is marked by the arrow.

should be stressed that in the $(\mathrm{EuO})_{2} /(\mathrm{BaO})_{2}$ superlattice the unit cell consists of four formula units and therefore four in-plane polarized polar phonons of $E_{u}$ symmetry can be expected in the IR spectra (two from the $\mathrm{EuO}$ and two from the $\mathrm{BaO}$ layers). The temperature dependence of the $\mathrm{EuO}$ optical phonon frequencies are shown in Fig. 8 for the nominally unstrained $\mathrm{EuO} / \mathrm{YSZ}$ from Fig. 2, the $+2.2 \%$ strained $\mathrm{EuO} / \mathrm{YAlO}_{3}$ from Fig. 3, and the $+6.4 \%$ strained $\left[(\mathrm{EuO})_{2} /(\mathrm{BaO})_{2}\right]_{35}$ superlattice from Fig. 5. The ferroelectric phase transition at $100 \mathrm{~K}$ is evident only in the most strained sample. DFT calculations support this observation and predict an in-plane ferroelectric polarization between 0.6 and $10 \mu \mathrm{C} / \mathrm{cm}^{2}$ (see Fig. 4 ) in the $+6.4 \%$ strained $\left[(\mathrm{EuO})_{2} /(\mathrm{BaO})_{2}\right]_{35}$ superlattice, but we could not measure it due to the electrically

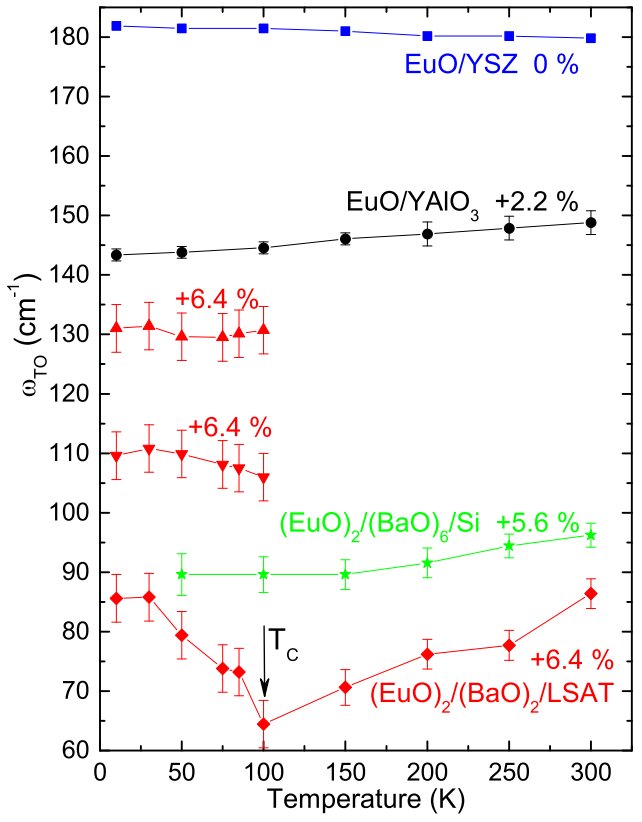

Fig. 8 Temperature dependence of the EuO optical phonon frequency in epitaxial (001)-oriented EuO films with various nominal strain levels imposed by underlying (001) YSZ, (110) $\mathrm{YAIO}_{3},(001) \mathrm{Si}$, and (001) LSAT substrates. $+5.6 \%$ and $+6.4 \%$ nominal tensile strain was reached in $\left[(\mathrm{EuO})_{2} /(\mathrm{BaO})_{6}\right]_{28} / \mathrm{Si}$ and $\left[(\mathrm{EuO})_{2} /(\mathrm{BaO})_{2}\right]_{35} /$ LSAT superlattices, respectively. The EuO phonon in the superlattice with the highest strain (+6.4\%) exhibits softening typical for a displacive ferroelectric phase transition. Upon undergoing the ferroelectric phase transition, the single optical phonon splits into three phonons due to the reduction of the symmetry of the $(\mathrm{EuO})_{2}$ unit cell that occurs below $T_{\mathrm{C}}$. The error bars for EuO/YSZ are comparable to the size of the symbols, but they increase with strain due to the reduced film thicknesses.

leaky a-Si capping of the superlattice. Ferroelectric hysteresis loops are traditionally used as evidence of being able to electrically switch the spontaneous polarization, although care is needed in concluding ferroelectricity from hysteresis loops ${ }^{39,40}$. In strained EuO, observation of the phonon anomaly near $100 \mathrm{~K}$ (Fig. 8) and the simultaneous peak in the dielectric permittivity (Fig. 7) cannot be explained by any other mechanism than a ferroelectric phase transition. Moreover, our observation is supported by the DFT calculations predicting the soft mode anomaly, the polar space group $\left(P m n 2_{1}\right)$, and the size of the spontaneous polarization (Fig. 4). Demonstrating that this spontaneous polarization in highly strained $\mathrm{EuO}$ can be switched by the application of an electric field awaits future studies using, for example, pyroelectric current or piezoelectric force microscopy measurements at low temperatures, provided the system can be prepared in a more insulating state.

Because of the technological relevance of silicon, we next studied $(\mathrm{EuO})_{x} /(\mathrm{BaO})_{y}$ superlattices on silicon substrates, which nominally impose a strain of $+5.6 \%$ in the EuO layers and $-1.6 \%$ in the $\mathrm{BaO}$ layers. To balance the opposing stresses in the $\mathrm{EuO}$ and $\mathrm{BaO}$ layers so that the in-plane spacing of the superlattice would approximately match to the underlying silicon substrate, we prepared a superlattice consisting of six $\mathrm{BaO}$ monolayers alternating with two $\mathrm{EuO}$ monolayers on (001) $\mathrm{Si}$ and repeated this $(\mathrm{EuO})_{2} /(\mathrm{BaO})_{6}$ stack 28 times. The total thickness of $\mathrm{BaO}$ in the $\left[(\mathrm{EuO})_{2} /(\mathrm{BaO})_{6}\right]_{28}$ superlattice was $46 \mathrm{~nm}$, while that of the $\mathrm{EuO}$ was $15 \mathrm{~nm}$. This allowed us to see both $\mathrm{BaO}$ and $\mathrm{EuO}$ phonons in the reflectance spectra (Fig. 9). We measured around ten different $(\mathrm{EuO})_{2} /(\mathrm{BaO})_{6}$ superlattice samples grown on silicon substrates with various resistivities. When the resistivity was high 


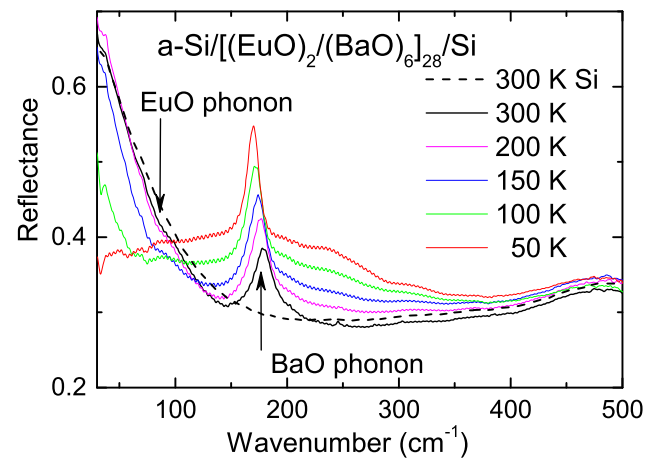

Fig. 9 IR reflectance measured at various temperatures of a [(EuO $\left.)_{2}\right]$

$\left.(\mathrm{BaO})_{6}\right]_{28}$ superlattice. The film with a total thickness of $61 \mathrm{~nm}$ was grown on a (001) Si substrate and capped with amorphous silicon. The spectrum of the bare silicon substrate at room temperature with a plasma reflectivity below $200 \mathrm{~cm}^{-1}$ is shown by the dashed line for comparison.

$(\rho>0.06 \Omega \cdot \mathrm{cm})$, the substrate was transparent in the far-IR region and we observed only interferences in the spectra due to multiple reflection from the top and back sides of the substrate and no signal from phonons in the $(\mathrm{EuO})_{2} /(\mathrm{BaO})_{6}$ superlattice was detected. If the substrate was optimally conducting $(0.01<\rho<0.04 \Omega \mathrm{cm})$, it was opaque in the far-IR region and the reflectivity from the free-carrier plasma was observed below $200 \mathrm{~cm}^{-1}$ (Fig. 9). The $\mathrm{BaO}$ phonon is seen as the sharp reflection peak in the spectra, while the $\mathrm{EuO}$ phonon is visible as a shallow minimum near $100 \mathrm{~cm}^{-1}$, since it lies in the range of the plasma edge of the substrate. The resistivity of the silicon substrate increases on cooling, so the plasma edge decreases and it finally disappears from the spectra below $50 \mathrm{~K}$. Below $30 \mathrm{~K}$, the substrate becomes transparent, interferences appear (not shown) and the spectrum is no longer sensitive to phonons in the superlattice. For that reason, we present spectra only above $50 \mathrm{~K}$ in Fig. 9, where the phonons can be identified.

In Fig. 10 we show the complex dielectric spectra of $\mathrm{EuO}$ and $\mathrm{BaO}$ in $\mathrm{a}\left[(\mathrm{EuO})_{2} /(\mathrm{BaO})_{6}\right]_{28}$ superlattice grown on silicon. We see that the $\mathrm{BaO}$ optical phonon frequency softens on cooling from $176 \mathrm{~cm}^{-1}$ to $169 \mathrm{~cm}^{-1}$ (see the peaks in the $\varepsilon^{\prime \prime}(\omega)$ spectra in Fig. 10b), and the EuO phonon softens from 94 to $87 \mathrm{~cm}^{-1}$ (see Figs. 8, 9, and 10). Although its frequency is much softer than in the unstrained thin film, it does not exhibit the anomaly that would be expected at a ferroelectric phase transition. We conclude, therefore, that the $+5.6 \%$ strain in $\left[(\mathrm{EuO})_{2} /(\mathrm{BaO})_{6}\right]_{28}$ superlattices appears to be insufficient to induce ferroelectricity in $\mathrm{EuO}$, consistent with our theoretical predictions in Fig. 3. Again consistent with the calculations, we note a much higher static relative permittivity $\left(\varepsilon_{0}^{\prime}=100-150\right)$ in the EuO component of the superlattice than in the unstrained $\mathrm{EuO}$ film $\left(\varepsilon_{0}^{\prime}=25-50\right.$ depending on the temperature) or in bulk $\mathrm{EuO}\left(\varepsilon_{0}^{\prime}=24\right)^{29}$, caused by the softening of the $\mathrm{EuO}$ phonon in the superlattice grown on $\mathrm{Si}$. This is a consequence of the Lyddane-Sachs-Teller relation $^{41}$. A strain-induced $50 \%$ increase of permittivity was recently also observed in $\mathrm{EuO} / \mathrm{LaAlO}_{3}$, where the $\mathrm{EuO}$ film was under $3 \%$ tensile strain ${ }^{42}$.

We also note that, while bulk $\mathrm{BaO}$ has an optical phonon frequency of $144 \mathrm{~cm}^{-1} 36$, our $\mathrm{BaO}$ layers in the $\left[(\mathrm{EuO})_{2} /\right.$ $\left.(\mathrm{BaO})_{6}\right]_{28}$ superlattice have a phonon frequency of $176 \mathrm{~cm}^{-1}$ (at room temperature), as expected for the compressive strain of $-1.6 \%$ (compressive strain causes hardening of the in-planepolarized $E_{u}$ phonon). As a result, the static relative permittivity of the $\mathrm{BaO}$ layers is lower than that of the EuO layers (see the low-frequency part of Fig. 10a). The $E_{u}$ phonon exhibits a small $\left(6 \mathrm{~cm}^{-1}\right)$ softening on cooling (see the shift to a lower frequency

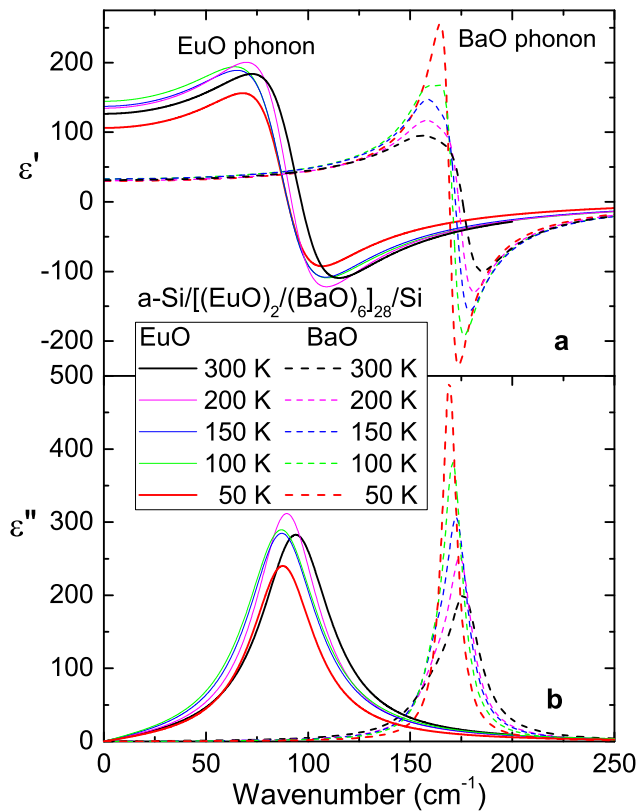

Fig. 10 Complex dielectric response of the $\mathrm{EuO}$ and $\mathrm{BaO}$ layers in the $\left[(\mathrm{EuO})_{2} /(\mathrm{BaO})_{6}\right]_{28}$ superlattice grown on a (001) Si substrate. Real and imaginary parts of the complex dielectric spectra are shown in panels (a) and (b), respectively. The spectra of the EuO (solid lines, nominal tensile strain $+5.6 \%$ ) and $\mathrm{BaO}$ (dashed lines, nominal compressive strain -1.6\%) layers were obtained from fits of the IR spectra in Fig. 9. Both optical phonons soften slightly on cooling, but they do not show any ferroelectric anomaly.

of the peak in the dielectric loss spectra $\varepsilon^{\prime \prime}(\omega)$ of $\mathrm{BaO}$ with reduced temperature) indicating a tendency toward a lattice instability. Since $\mathrm{BaO}$ is under compressive strain, however, larger softening can be expected for the $A_{2 \mathrm{u}}$ symmetry phonon polarized perpendicularly to the sample plane, but this phonon is not activated in the near-normal IR reflectivity geometry employed.

Magnetic properties of $(\mathrm{EuO})_{x} /(\mathrm{BaO})_{y}$ superlattices. Having established that it is possible to use strain to induce ferroelectricity in $(\mathrm{EuO})_{x} /(\mathrm{BaO})_{y}$ superlattices, we now turn to the question of whether such superlattices are simultaneously ferroelectric and ferromagnetic, i.e., multiferroic. Bulk $\mathrm{EuO}$ is ferromagnetic below $69 \mathrm{~K}^{43}$. It was reported that the ferromagnetic Curie temperature $\left(T_{\mathrm{C}}\right)$ of thick films of unstrained (001) EuO / (001) YSZ is the same as that of bulk EuO, but that it reduces in films with thickness less than $10 \mathrm{~nm}^{44}$. First-principles calculations suggest that biaxial tension will reduce the ferromagnetic $T_{\mathrm{C}}$ of $\mathrm{EuO}^{45}$ and consistent with this prediction tensile strained (001) $\mathrm{EuO}$ films grown on (110) $\mathrm{LuAlO}_{3}$ show a reduced ferromagnetic $T_{\mathrm{C}}{ }^{44}$. Thus, we expect the ferromagnetic $T_{\mathrm{C}}$ of strained $(\mathrm{EuO})_{x} /(\mathrm{BaO})_{y}$ superlattices to be lower than bulk $\mathrm{EuO}$ due to the reduced dimensionality and tensile strain on the EuO layers in the $(\mathrm{EuO})_{x} /(\mathrm{BaO})_{y}$ superlattices. Intermixing is also expected to decrease the ferromagnetic $T_{C}$ due to the dilution of the ferromagnetic $\mathrm{Eu}^{2+}$ spins with diamagnetic $\mathrm{Ba}^{2+}$ spins as demonstrated by $\mathrm{Eu}_{x} \mathrm{Sr}_{1-x} \mathrm{O}$ being no longer ferromagnetic for $x<0.136$ (the 3D percolation limit of an FCC lattice of diluted spins) ${ }^{46}$.

We measured the temperature dependence of the magnetic susceptibility of $(\mathrm{EuO})_{x} /(\mathrm{BaO})_{y}$ superlattices grown on various substrates to determine the ferromagnetic $T_{\mathrm{C}}$ of each superlattice (Fig. 11 and Supplementary Figs. 5 and 6). Importantly, all of the superlattices exhibited ferromagnetic hysteresis loops at low temperatures, even down to a single monolayer thickness of the $\mathrm{EuO}$ (e.g., in $\left[(\mathrm{EuO})_{1} /(\mathrm{BaO})_{4}\right]_{26} / \mathrm{Si}$-see Supplementary Fig. 6). In addition, one can see two main trends. First, the ferromagnetic $T_{\mathrm{C}}$ 


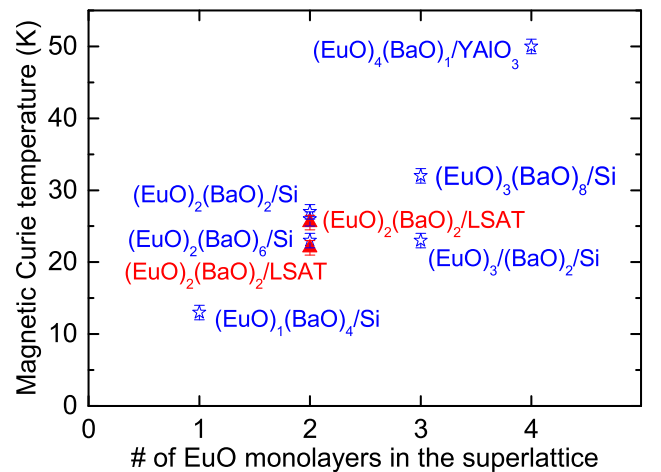

Fig. 11 Ferromagnetic Curie temperatures of $(\mathrm{EuO})_{x} /(\mathrm{BaO})_{y}$ superlattices grown on various substrates. The error bars of these $T_{C} S$ are shown and are only slightly larger than the symbols.

rises with increasing thickness of the EuO layers. Second, the ferromagnetic $T_{\mathrm{C}}$ decreases with increasing tensile strain in the $\mathrm{EuO}$ layers and increasing thickness of the $\mathrm{BaO}$ layers. These trends are all expected, due to the reduction of the exchange coupling between intralayer and interlayer $\mathrm{Eu}^{2+}$ spins. The two $(\mathrm{EuO})_{2} /(\mathrm{BaO})_{2}$ superlattices on (001) LSAT exhibit slightly different $T_{\mathrm{C}}$ values, likely due to differences in sample quality including slight strain relaxation, but more importantly both samples show ferromagnetism above $20 \mathrm{~K}$.

Based on the IR and magnetic data presented above, we conclude that $(\mathrm{EuO})_{2} /(\mathrm{BaO})_{2}$ superlattices strained to $(001)$ LSAT exhibit a combination of simultaneous ferromagnetism and ferroelectricity, i.e., they exhibit the exact definition of multiferroism, in contrast to most magnetic ferroelectrics which combine antiferromagnetic and ferroelectric order. This makes these EuO-based multiferroic superlattices not only the simplest known multiferroic, but also the one exhibiting the highest temperature for this strong multiferroic state (simultaneous ferromagnetism plus ferroelectricity $)^{14}$. The magnetoelectric coupling should be higher in $(\mathrm{EuO})_{2} /(\mathrm{BaO})_{2}$ superlattices than in ferroelectric antiferromagnets ${ }^{47}$. Unfortunately, we could not directly measure the ferroelectric hysteresis loops and magnetoelectric coupling, because the superlattices had an a-Si cap (to protect them against degradation when exposed to air), which was rather electrically leaky and it prevented application of an electric field essential for the magnetoelectric studies.

A strain-induced displacive ferroelectric instability is rather surprising in ferromagnetic EuO because the permittivity and phonon frequencies are almost temperature independent in bulk $\mathrm{EuO}$. Strain-induced ferroelectric phase transitions have so far been reported mainly in incipient ferroelectrics like the perovskites $\mathrm{SrTiO}_{3}{ }^{48}, \mathrm{KTaO}_{3}{ }^{49}, \mathrm{EuTiO}_{3}{ }^{14}$, and $\mathrm{NaMnF}_{3}{ }^{9}$ or in related $\mathrm{Sr}_{n+1} \mathrm{Ti}_{n} \mathrm{O}_{3 n}{ }^{50}$ phases with Ruddlesden-Popper structures. In all of these bulk systems, the permittivity and soft phonons driving the ferroelectric phase transition are strongly temperature dependent and therefore they are very sensitive to $\operatorname{strain}^{14,49-52}$. For that reason, the ferroelectric phase transition can be induced in these originally paraelectric (sometimes called quantum paraelectric) materials. Interestingly, a strain-induced ferroelectric phase transition has been never observed in a simple binary monoxide ( $\mathrm{XO}$ with $\mathrm{X}=\mathrm{Ba}, \mathrm{Sr}, \mathrm{Eu}, \mathrm{Ca}$, etc.) with the rocksalt crystal structure, although it was theoretically predicted in refs. ${ }^{7,20}$. The reason is the very high strain expected for the soft-mode-driven ferroelectric phase transition in $\mathrm{XO}$. This is because the phonons in monoxides have much higher frequencies than in perovskites and therefore they are usually very stable with temperature. In this report, we have experimentally confirmed
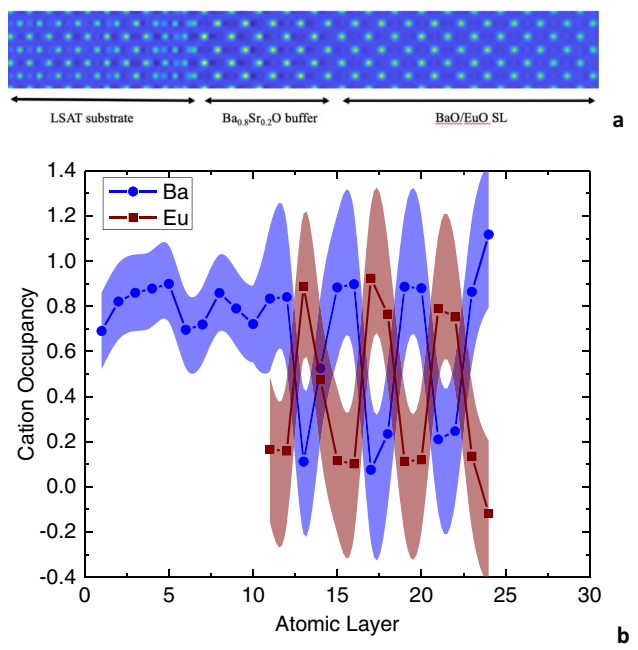

Fig. 12 Structural characterization at room temperature of a $(\mathrm{EuO})_{2} /$ $(\mathrm{BaO})_{2}$ superlattice grown on a (001) LSAT substrate. a COBRA analysis of the electron density in the (110) plane. b Cation occupancy of a (EuO) $)_{2}$ $(\mathrm{BaO})_{2}$ superlattice. It was grown on top of a 5 -unit-cell-thick $(2.7 \mathrm{~nm})$ $\mathrm{Ba}_{0.8} \mathrm{Sr}_{0.2} \mathrm{O}$ buffer layer on a (001) LSAT substrate.

that in $\mathrm{EuO}$ more than $6 \%$ strain is required to induce a phonon instability producing a displacive ferroelectric phase transition.

In summary, we have prepared epitaxial $\mathrm{EuO}$ thin films and $(\mathrm{EuO})_{x} /(\mathrm{BaO})_{y}$ superlattices in which the $\mathrm{EuO}$ is strained up to nominally $+6.4 \%$. For this largest strain, which was achieved on a $\left[(\mathrm{EuO})_{2} /(\mathrm{BaO})_{2}\right]_{35}$ superlattice grown on (001) LSAT, our phonon spectra reveal the hallmark of a ferroelectric transition around $100 \mathrm{~K}$, i.e., phonon softening and a peak in permittivity around this temperature. These strained superlattices retain their bulk ferromagnetism, i.e., they are multiferroic. The qualitative picture is consistent with previous DFT-GGA calculations, but the quantitative values are in better agreement with the B1WC hybrid functional.

\section{Methods}

Molecular-beam epitaxial growth of strained EuO films and (EuO) $x /(\mathrm{BaO})_{y}$ superlattices. The films and superlattices were grown in Veeco GEN10 and 930 oxide MBE systems on various single crystalline substrates which allowed us to impose different strains via heteroepitaxy. In the absence of relaxation (i.e., fully commensurate films), EuO films grown on cubic (001) yttria-stabilized zirconia $\mathrm{ZrO}_{2}$ (YSZ) have $0 \%$ strain, on $(110) \mathrm{YAlO}_{3}$ have $+2.2 \%$ strain and on $(001) \mathrm{Si}$ have $+5.6 \%$ strain. In commensurate $(\mathrm{EuO})_{2} /(\mathrm{BaO})_{2}$ superlattices grown on $(001)$ $\left(\mathrm{LaAlO}_{3}\right)_{0.29}-\left(\mathrm{SrAl}_{1 / 2} \mathrm{Ta}_{1 / 2} \mathrm{O}_{3}\right)_{0.71}$ (LSAT) substrates the $\mathrm{EuO}$ monolayers are under $+6.4 \%$ strain at the same time that the $\mathrm{BaO}$ monolayers are under $-1.2 \%$ strain. All of the above strains are biaxial in-plane strains; our objective is to induce an inplane ferroelectric polarization, which can be revealed by observing a softening of the polar phonon driving the phase transition by IR spectroscopy.

The structural quality of the biaxially strained $\mathrm{EuO}$ films and $(\mathrm{EuO})_{x} /(\mathrm{BaO})_{y}$ superlattices was assessed by reflection high-energy electron diffraction (RHEED) during growth and XRD after growth (see Supplementary Fig. 1-4). Although epitaxial EuO films have been imaged by transmission electron microscopy $(\mathrm{TEM})^{53-57}$, attempts to image these highly strained $(\mathrm{EuO})_{x} /(\mathrm{BaO})_{y}$ superlattices by TEM were unsuccessful.

Coherent Bragg rod analysis (COBRA) at room temperature was used to characterize the most strained structure, a $(\mathrm{EuO})_{2} /(\mathrm{BaO})_{2}$ superlattice grown on (001) LSAT. The resulting electron density analysis is shown in Fig. 12 at atomic resolution. The presence of the 5-unit-cell-thick $(2.7 \mathrm{~nm}) \mathrm{Ba}_{0.8} \mathrm{Sr}_{0.2} \mathrm{O}$ epitaxial buffer layer on top of the (001) LSAT substrate is evident. The low growth temperature $\left(80^{\circ} \mathrm{C}\right)$ used for the growth of this superlattice resulted in minimal intermixing between the $\mathrm{EuO}$ and $\mathrm{BaO}$ layers. An analysis of cation occupancy shows that there is about $15 \%$ intermixing between the europium and barium cation sites in the superlattice. Note that this intermixing is the combination of interface roughness and interdiffusion. We believe most of the indicated site intermixing arises from the former mechanism, i.e., is due to the presence of atomic steps at the interface between the $\mathrm{BaO}$ and EuO layers. This is because the superlattice diffraction peaks in XRD (not shown) do not wash out until the growth temperature reaches about $150^{\circ} \mathrm{C}$. 
Since $\mathrm{EuO}$ is reactive with the humidity in air, the films were always protected by an a-Si cap with a thickness of about 100-300 $\mathrm{nm}$. This capping layer has no IRactive optical phonons, so does not noticeably influence the spectra. We found experimentally by RHEED and XRD investigations (not shown) that, with increasing strain, films and superlattices must be grown at decreasing temperatures to avoid relaxation. This required growth temperatures as low as $\sim 120^{\circ} \mathrm{C}$ for growth on silicon and $\sim 80^{\circ} \mathrm{C}$ for growth on LSAT substrates. At these temperatures, EuO cannot be grown using the typical adsorption-controlled growth method ${ }^{58}$ in which nearly stoichiometric films form readily within a broad range of oxygen pressure because the film stoichiometry is controlled by thermodynamics. Instead, at the low growth temperatures used, the fluxes of the cation species (europium and barium) have to be matched precisely to each other and to the oxygen flux at the sample location. Otherwise phases exhibiting other oxidation states than the desired phases form leading to quick relaxation of the superlattices. The impurity phases can be detected with high sensitivity by observation of diffraction maxima in RHEED and after prolonged growth also in XRD. To achieve films and superlattices with no extra diffraction maxima in RHEED and XRD required the oxygen partial pressure to be controlled with a precision better than $\pm 2 \%$ using a residual gas analyzer (at a base oxygen partial pressure of (1-5) $\times 10^{-9}$ Torr) and meticulous calibration of the metal fluxes before each growth cycle.

Before superlattice growth on silicon, we heated the silicon wafers to $\approx 900^{\circ} \mathrm{C}$ for $5 \mathrm{~min}$ to thermally desorb the native $\mathrm{SiO}_{2}$-layer on the substrate. Subsequently, the wafer was cooled to a temperature of $650^{\circ} \mathrm{C}$ and a monolayer of strontium metal was deposited under high-vacuum conditions. Later, this layer was oxidized at an oxygen background partial pressure of $\approx 3 \times 10^{-8}$ Torr and a substrate temperature of $190^{\circ} \mathrm{C}$. This intermediate step is suitable to prevent oxidation of the silicon substrate during the first stages of oxide growth and provides a template layer for the epitaxial growth of overlying oxides.

Superlattice growth on silicon started with the deposition of three unit cells of $\mathrm{BaO}$ at a substrate temperature of $90^{\circ} \mathrm{C}$, followed by the deposition of 1 unit cell of $\mathrm{EuO}$. Note that a unit cell of the $A \mathrm{O}$ rocksalt structure, where $A=\mathrm{Ba}$ or Eu in the current study, contains two $A \mathrm{O}$ monolayers. After the deposition of one of these superlattice unit cells, the substrate temperature was increased to the final growth temperature of $\sim 125^{\circ} \mathrm{C}$. The lower deposition temperature for $\mathrm{BaO} / \mathrm{Si}$ was found to prevent relaxation of the initial $\mathrm{BaO}$ layer, whereas a higher temperature was needed to ensure high-quality epitaxial growth of thicker superlattices $(>50 \mathrm{~nm})$.

At growth temperatures $>>100^{\circ} \mathrm{C}, \mathrm{BaO}$ relaxes after the deposition of $<3$ monolayers (giving rise to extra spots in RHEED diffraction patterns). This is only a problem during deposition of the first few monolayers of the whole superlattice and probably due to weaker bonding between the SrO-covered silicon substrate and $\mathrm{BaO}$ as compared with the $\mathrm{BaO} / \mathrm{BaO}$ or $\mathrm{BaO} / \mathrm{EuO}$ bonding strength. The critical thickness for the relaxation of EuO on silicon at a growth temperature of $\sim 100^{\circ} \mathrm{C}$ was determined to be $\sim 1$ monolayer.

Therefore, deposition was started with $\mathrm{BaO}$ at temperatures below $100^{\circ} \mathrm{C}$ (typically $95^{\circ} \mathrm{C}$ ) and after the deposition of $\sim 2$ monolayers, the substrate temperature was gradually increased during deposition of the following $\sim 10$ monolayers of the superlattice to the final growth temperature of $\sim 125^{\circ} \mathrm{C}$.

Magnetic studies. The magnetic properties of the $(\mathrm{EuO})_{x} /(\mathrm{BaO})_{y}$ superlattices were investigated using a Quantum Design (MPMS) superconducting quantum interference device with magnetic fields up to $5 \mathrm{~T}$. Results of temperature and magnetic field measurements of magnetization are shown in Supplementary Figs. 5 and 6 , respectively.

\section{Infrared spectroscopy and determination of phonon parameters. The IR} reflectance experiments were performed using a Bruker IFS 113v Fourier-transform IR spectrometer equipped with a helium-cooled $(1.6 \mathrm{~K})$ silicon bolometer. The polarized reflectance measurements were realized in a near-normal incidence geometry sensitive only to in-plane polarized phonons. Bare substrates and films upon substrates were measured under the same conditions on cooling from room temperature to $10 \mathrm{~K}$ in an Optistat CF cryostat (Oxford Instruments). The 3-mmthick polyethylene windows used in the cryostat limited the measured spectra range at low temperatures because the windows are IR transparent only up to $650 \mathrm{~cm}^{-1}$. Room-temperature spectra were detected up to $3000 \mathrm{~cm}^{-1}$ using a pyroelectric detector made of deuterated triglicine sulfate.

To evaluate the IR reflectance spectra, a model corresponding to a two-layer optical system was employed for fitting of EuO films on $\mathrm{YSZ}$ or $\mathrm{YAlO}_{3}$ substrates. $(\mathrm{EuO})_{x} /(\mathrm{BaO})_{y}$ superlattices with $n$ repetitions were approximated by $\mathrm{EuO}$ and $\mathrm{BaO}$ films with thickness $n . d_{i}$, where $d_{i}$ denotes the thickness of the $(\mathrm{EuO})_{x}$ and $(\mathrm{BaO})_{y}$ layer $(i=x, y)$. In this case, a model for the three-layer optical system $\mathrm{EuO} /$ $\mathrm{BaO} /$ substrate was employed to fit the spectra. Details of this model are described in ref. ${ }^{59}$ and in Supplementary Information. We first fit the IR reflectivity spectra of the bare substrate at various temperatures. In our experiment, the reflectivity $R$ $(\omega)$ is related to the complex dielectric function $\varepsilon^{*}(\omega)$ by

$$
\boldsymbol{R}(\omega)=\left|\frac{\sqrt{\varepsilon^{*}}-1}{\sqrt{\varepsilon^{*}}+1}\right|^{2} .
$$

The complex permittivity spectra of the substrates were described by a generalized, factorized damped harmonic oscillator model

$$
\varepsilon^{*}(\omega)=\varepsilon^{\prime}(\omega)+i \varepsilon^{\prime \prime}(\omega)=\varepsilon_{\infty} \prod_{j} \frac{\omega_{\mathrm{LO} j}^{2}-\omega^{2}+\mathrm{i} \omega \gamma_{\mathrm{LO} j}}{\omega_{\mathrm{TO} j}^{2}-\omega^{2}+\mathrm{i} \omega \gamma_{\mathrm{TO} j}},
$$

where $\omega_{\mathrm{TO} j}$ and $\omega_{\mathrm{LO} j}$ are the frequencies of the $j$ th transverse optic and longitudinal optic phonons, and $\gamma_{\mathrm{TO} j}$ and $\gamma_{\mathrm{LO} j}$ are the corresponding damping constants. $\varepsilon_{\infty}$ is the high-frequency (electronic) contribution to the permittivity, determined from the room-temperature frequency-independent reflectivity tail above the phonon frequencies. The dielectric function of each thin films has the form of a sum of $N$ independent three-parameter damped harmonic oscillators (representing the inplane polarized transverse optic phonons of the film), which are expressed as

$$
\varepsilon^{*}(\omega)=\varepsilon_{\infty}+\sum_{j=1}^{N} \frac{\Delta \varepsilon_{j} \omega_{\mathrm{TO} j}^{2}}{\omega_{\mathrm{TO} j}^{2}-\omega^{2}+\mathrm{i} \omega \gamma_{\mathrm{TO} j}},
$$

where $\Delta \varepsilon_{j}$ is the dielectric strength of the jth mode. In the paraelectric $\mathrm{EuO}$ and $\mathrm{BaO}$ films, only a single polar optic phonon is IR active (i.e., $N=1$ ), but in $(\mathrm{EuO})_{2} /$ $(\mathrm{BaO})_{2} / \mathrm{LSAT}$ two additional phonons (i.e., $N=3$ ) activate in the IR spectra below $T_{\mathrm{C}}=100 \mathrm{~K}$ due to the lowering of the crystal symmetry that accompanies the ferroelectric phase transition. Equation (3) is simpler than Eq. (2), but it is well justified because the damping of the longitudinal phonons of the films does not influence the reflectance spectra appreciably.

First-principles calculations. For calculations of the phonons in $\mathrm{EuO}$, the hybridfunctional B1WC ${ }^{32}$ implemented in the CRYSTAL17 code was used ${ }^{33}$. We used pseudopotentials for Ba (Ba_HAYWSC-3111(2d)G_zagorac_2012) ${ }^{60}$ and Eu (Eu (II)_ECP53MWB-VTZ_Dolg, with the $4 f$-electrons treated as core electrons $)^{61}$ and an all-electron basis for $\mathrm{O}^{62}$. A grid of k-point of 8 was used for the in-plane directions and 1 along the out-of-plane direction when the thickness is larger than 4 atomic layers and 2 otherwise (no calculation was done with a thickness smaller than 4 monolayers). The phonons were calculated by the finite difference method based on the numerical calculation of the second derivatives of the total energy, as reported in ref. ${ }^{63}$.

\section{Data availability}

All of the relevant data are available from the corresponding author S.K. (kamba@fzu.cz) upon reasonable request.

\section{Code availability}

The first-principles calculations were carried out using the proprietary CRYSTAL17 code (www.crystal.unito.it). The visualization software VESTA is distributed free of charge for scientific users under the VESTA license (https://jpminerals.org/vesta/en/download. html).

Received: 14 July 2020; Accepted: 21 September 2020; Published online: 14 October 2020

\section{References}

1. Dawber, M., Rabe, K. M. \& Scott, J. F. Physics of the thin-film ferroelectric oxides. Rev. Mod. Phys. 77, 1083-1130 (2005).

2. Schlom, D. G. et al. Strain tuning of ferroelectric thin films. Annu. Rev. Mater. Res. 37, 589-626 (2007).

3. Schlom, D. G. et al. Elastic strain engineering of ferroic oxides. MRS Bull. 39, 118-130 (2014).

4. Agar, J. C. et al. Frontiers in strain-engineered multifunctional ferroic materials. MRS Commun. 6, 151-166 (2016).

5. Fennie, C. J. \& Rabe, K. M. Magnetic and electric phase control in epitaxial $\mathrm{EuTiO}_{3}$ from first principles. Phys. Rev. Lett. 97, 267602 (2006).

6. Lee, J. H. \& Rabe, K. M. Epitaxial-strain-induced multiferroicity in $\mathrm{SrMnO}_{3}$ from first principles. Phys. Rev. Lett. 104, 207204 (2010).

7. Bousquet, E., Spaldin, N. A. \& Ghosez, P. Strain-induced ferroelectricity in simple rocksalt binary oxides. Phys. Rev. Lett. 104, 037601 (2010).

8. Garcia-Castro, A. C., Romero, A. H. \& Bousquet, E. Strain-engineered multiferroicity in Pnma $\mathrm{NaMnF}_{3}$ fluoroperovskite. Phys. Rev. Lett. 116, 117202 (2016).

9. Yang, M. et al. Room temperature ferroelectricity in fluoroperovskite thin films. Sci. Rep. 7, 7182 (2017).

10. Wojdel, J. C. \& Iniguez, J. Magnetoelectric response of multiferroic $\mathrm{BiFeO}_{3}$ and related materials from first-principles calculations. Phys. Rev. Lett. 103, 267205 (2009).

11. Wojdel, J. C. \& Iniguez, J. Ab Initio indications for giant magnetoelectric effects driven by structural softness. Phys. Rev. Lett. 105, 037208 (2010). 
12. Bousquet, E. \& Spaldin, N. Induced magnetoelectric response in Pnma perovskites. Phys. Rev. Lett. 107, 197603-197605 (2011).

13. Halley, D. et al. Size-induced enhanced magnetoelectric effect and multiferroicity in chromium oxide nanoclusters. Nat. Commun. 5, 3167 (2014).

14. Lee, J. H. et al. A strong ferroelectric ferromagnet created by means of spinlattice coupling. Nature 466, 954-959 (2010).

15. Bhattacharjee, S., Bousquet, E. \& Ghosez, P. Engineering multiferroism in $\mathrm{CaMnO}_{3}$. Phys. Rev. Lett. 102, 117602 (2009).

16. Günter, T. et al. Incipient ferroelectricity in $2.3 \%$ tensile-strained $\mathrm{CaMnO}_{3}$ films. Phys. Rev. B 85, 214120 (2012)

17. Becher, C. et al. Strain-induced coupling of electrical polarization and structural defects in $\mathrm{SrMnO}_{3}$ films. Nat. Nanotechnol. 10, 661-665 (2015).

18. Kamba, S. et al. Strong spin-phonon coupling in infrared and Raman spectra of $\mathrm{SrMnO}_{3}$. Phys. Rev. B 89, 064308 (2014).

19. Maurel, L. et al. Nature of antiferromagnetic order in epitaxially strained multiferroic $\mathrm{SrMnO}_{3}$ thin films. Phys. Rev. B 92, 024419 (2015).

20. Kim, B. G. Epitaxial strain induced ferroelectricity in rocksalt binary compound: Hybrid functional $\mathrm{Ab}$ initio calculation and soft mode group theory analysis. Sol. State Commun. 151, 674-677 (2011).

21. Suits, J. C. \& Lee, K. Giant magneto-optical kerr effect in EuO. J. Appl. Phys. 42, 3258-3260 (1971).

22. Matsubara, M. et al. Ultrafast optical tuning of ferromagnetism via the carrier density. Nat. Commun. 6, 6724 (2015).

23. Shapira, Y., Foner, S. \& Reed, T. B. EuO. I. Resistivity and Hall effect in fields up to 150 kOe. Phys. Rev. B 8, 2299-2315 (1973).

24. Averyanov, D. V. et al. Fine structure of metal-insulator transition in EuO resolved by doping engineering. Nanotechnology 29, 195706 (2018).

25. Yamasaki, T., Ueno, K., Tsukazaki, A., Fukumura, T. \& Kawasaki, M. Observation of anomalous Hall effect in EuO epitaxial thin films grown by a pulse laser deposition. Appl. Phys. Lett. 98, 082116 (2011).

26. Melville, A. et al. Lutetium-doped EuO films grown by molecular-beam epitaxy. Appl. Phys. Lett. 100, 222101 (2012).

27. Schmehl, A. et al. Epitaxial integration of the highly spin-polarized ferromagnetic semiconductor $\mathrm{EuO}$ with silicon and GaN. Nat. Mater 6, 882 (2007).

28. Petzelt, J. \& Kamba, S. Far infrared and terahertz spectroscopy of ferroelectric soft modes in thin films: a review. Ferroelectrics 503, 19-44 (2016).

29. Axe, J. D. Infrared dielectric dispersion in divalent europium chalcogenides. J. Phys. Chem. Solids 30, 1403-1406 (1969).

30. Diehl, R. \& Brandt, G. Crystal structure refinement of $\mathrm{YAlO}_{3}$, a promising laser material. Mater. Res. Bull. 10, 85-90 (1975).

31. Pradip, R. et al. Phonon confinement and spin-phonon coupling in tensilestrained ultrathin EuO films. Nanoscale 11, 10968-10976 (2019).

32. Bilc, D. I. et al. Hybrid exchange-correlation functional for accurate prediction of the electronic and structural properties of ferroelectric oxides. Phys. Rev. B 77, 165107 (2008).

33. Erba, A., Baima, J., Bush, I., Orlando, R. \& Dovesi, R. Large-scale condensed matter DFT simulations: performance and capabilities of the CRYSTAL code. J. Chem. Theory Comput. 13, 5019-5027 (2017).

34. Yang, X., Wang, Y., Yan, H. \& Chen, Y. Effects of epitaxial strains on spontaneous polarizations and band gaps of alkaline-earth-metal oxides MO $(\mathrm{M}=\mathrm{Mg}, \mathrm{Ca}, \mathrm{Sr}, \mathrm{Ba})$. Comput. Mater. Sci. 121, 61-66 (2016).

35. Galtier, M., Montaner, A. \& Vidal, G. Phonon optiques de $\mathrm{CaO}, \mathrm{SrO}, \mathrm{BaO}$ au centre de la zone de Brillouin a 300 et 17 K. J. Phys. Chem. Solids 33, 2295-2302 (1972).

36. Chang, S. S., Tompson, C. W., Gürmen, E. \& Muhlestein, L. D. Lattice dynamics of BaO. J. Phys. Chem. Solids 36, 769-773 (1975).

37. Matthews, J. W. \& Blakeslee, A. E. Defects in epitaxial multilayers. III. Preparation of almost perfect multilayers. J. Cryst. Growth 32, 265-273 (1976).

38. Goian, V. et al. Antiferrodistortive phase transition in $\mathrm{EuTiO}_{3}$. Phys. Rev. $B$ 86, 054112 (2012)

39. Scott, J. F. Ferroelectrics go bananas. J. Phys.: Condens. Matter 20, 021001 (2008).

40. Vasudevan, R. K., Balke, N., Maksymovych, P., Jesse, S. \& Kalinin, S. V. Ferroelectric or non-ferroelectric: Why so many materials exhibit "ferroelectricity" on the nanoscale. Appl. Phys. Rev. 4, 021302 (2017).

41. Lyddane, R. H., Sachs, R. G. \& Teller, E. On the polar vibrations of alkali halides. Phys. Rev. 59, 673-676 (1941).

42. Kashir, A., Jeong, H.-W., Jung, W., Jeong, Y. H. \& Lee, G.-H. Strain-Induced Increase of dielectric constant in EuO thin film. Mater. Res. Express 6, 106321 (2019).

43. McGuire, T. R. \& Shafer, M. W. Ferromagnetic europium compounds. J. Appl. Phys. 35, 984-988 (1964)

44. Melville, A. et al. Effect of film thickness and biaxial strain on the Curie temperature of EuO. Appl. Phys. Lett. 102, 062404 (2013).

45. Ingle, N. J. C. \& Elfimov, I. S. Influence of epitaxial strain on the ferromagnetic semiconductor EuO: first-principles calculations. Phys. Rev. B 77, 121202 (2008).
46. Stroka, B. et al. Specific heat of $\mathrm{Eu}_{x} \mathrm{Sr}_{1-x} \mathrm{O}$ near the ferromagnetic phase transition. Z. Phys. B - Condens. Matter 89, 39-43 (1992).

47. Eerenstein, W., Mathur, N. D. \& Scott, J. F. Multiferroic and magnetoelectric materials. Nature 442, 759-765 (2006).

48. Haeni, J. H. et al. Room-temperature ferroelectricity in strained $\mathrm{SrTiO}_{3}$. Nature 430, 758-761 (2004).

49. Skoromets, V. et al. Ferroelectric phase transition in polycrystalline $\mathrm{KTaO}_{3}$ thin film revealed by terahertz spectroscopy. Appl. Phys. Lett. 99, 052908 (2011).

50. Lee, C.-H. et al. Exploiting dimensionality and defect mitigation to create tunable microwave dielectrics. Nature 502, 532-536 (2013).

51. Nuzhnyy, D. et al. Soft mode behavior in $\mathrm{SrTiO}_{3} / \mathrm{DyScO}_{3}$ thin films: evidence of ferroelectric and antiferrodistortive phase transitions. App. Phys. Lett. 95, 232902 (2009)

52. Peng, W. W. et al. Room-temperature soft mode and ferroelectric like polarization in $\mathrm{SrTiO}_{3}$ ultrathin films: Infrared and ab initio study. Sci. Rep. 7, 2160 (2017)

53. Mundy, J. A. et al. Hetero-epitaxial EuO interfaces studied by analytic electron microscopy. Appl. Phys. Lett. 104, 091601 (2014).

54. Mairoser, T. et al. High-quality EuO thin films the easy way via topotactic transformation. Nat. Commun. 6, 7716 (2015)

55. Caspers, C. et al. Interface engineering to create a strong spin filter contact to silicon. Sci. Rep. 6, 22912 (2016).

56. Averyanov, D. V. et al. Atomic-scale engineering of abrupt interface for direct spin contact of ferromagnetic semiconductor with silicon. Sci. Rep. 6, 22841 (2016).

57. Averyanov, D. V. et al. Direct epitaxial integration of the ferromagnetic semiconductor $\mathrm{EuO}$ with Si(111). J. Magn. Magn. Mater. 459, 136-140 (2018).

58. Ulbricht, R. W., Schmehl, A., Heeg, T., Schubert, J. \& Schlom, D. G. Adsorption-controlled growth of EuO by molecular-beam epitaxy. Appl. Phys. Lett. 93, 102105 (2008).

59. Dressel, M. \& Grüner, G. Electrodynamics of Solids 416-420 (Cambridge Press 2002)

60. Zagorac, D., Doll, K., Schon, J. C. \& Jansen, M. Sterically active electron pairs in lead sulfide? An investigation of the electronic and vibrational properties of $\mathrm{PbS}$ in the transition region between the rock salt and the alpha-GeTe-type modifications. Chemistry 18, 10929-10936 (2012).

61. Yang, J. \& Dolg, M. Valence basis sets for lanthanide 4f-in-core pseudopotentials adapted for crystal orbital ab initio calculations. Theor. Chem. Accounts 113, 212-224 (2005).

62. Varignon, J. PhD thesis. https://tel.archives-ouvertes.fr/tel-00651567/ document.

63. Pascale, F. et al. The calculation of the vibrational frequencies of crystalline compounds and its implementation in the CRYSTAL Code. J. Comput. Chem. 25, 888-897 (2004)

\section{Acknowledgements}

V.G. (Prague) and S.K. were supported by the Czech Science Foundation (Project No. 18 09265 S) and by MŠMT Project No. SOLID21-CZ.02.1.01/0.0/0.0/16_019/0000760. R.H A.M., and D.G.S. acknowledge support from the National Science Foundation (Platform for Accelerated Realization, Analysis and Discovery of Interface Materials (PARADIM)) under Cooperative Agreement No. DMR-1539918 and by the Gordon and Betty Moore Foundation through Grant GBMF9073 to Cornell University. Sample preparation was in part facilitated by the Cornell NanoScale Facility, a member of the National Nanotechnology Coordinated Infrastructure (NNCI), which is supported by the National Science Foundation (Grant NNCI-1542081). This work made use of Cornell Center for Materials Research Shared Facilities, which are supported through the NSF MRSEC program (DMR-1719875). V.G. (Penn State) and Y.Y. acknowledge support from the Department of Energy grant number DE-SC0012375 for performing the COBRA work. This research used resources of the Advanced Photon Source, a U.S. Department of Energy (DOE) Office of Science User Facility operated for the DOE Office of Science by Argonne National Laboratory under Contract No. DE-AC02-06CH11357. E.B. thanks the FRSFNRS. N.A.S. acknowledges ETH Zürich and the Koerber foundation for funding support E.B. and Ph.G. acknowledge the ARC project AIMED, the F.R.S-FNRS PDR projects MaRePeThe and HiT4Fit, and the M-ERA.NET project SIOX for funding. E.B. and Ph.G. have relied on the PRACE project, the DeNoMo and on the Céci facilities funded by F.R.SFNRS (Grant No. 2.5020.1) and Tier-1 super-computer of the Federation WallonieBruxelles funded by the Walloon Region (Grant No. 1117545) for simulations.

\section{Author contributions}

V.G. (Prague) measured and evaluated IR spectra. S.K. conducted the IR studies and wrote the first version of the manuscript. R.H. and A.M., under the supervision of D.G.S synthesized the samples by MBE and characterized them by RHEED, XRD, and SQUID measurements. E.B, P.G., and N.A.S. performed first-principles density functional calculations. Y.Y. and H.Z. performed COBRA analysis. V.G. (Penn State) measured second harmonic generation in less strained films. All authors discussed results and commented on the manuscript. The study was conceived and guided by D.G.S. and S.K. 


\section{Competing interests}

The authors declare no competing interests.

\section{Additional information}

Supplementary information is available for this paper at https://doi.org/10.1038/s43246020-00075-1.

Correspondence and requests for materials should be addressed to S.K.

Reprints and permission information is available at http://www.nature.com/reprints

Publisher's note Springer Nature remains neutral with regard to jurisdictional claims in published maps and institutional affiliations. (c) (i) Open Access This article is licensed under a Creative Commons Attribution 4.0 International License, which permits use, sharing, adaptation, distribution and reproduction in any medium or format, as long as you give appropriate credit to the original author(s) and the source, provide a link to the Creative Commons license, and indicate if changes were made. The images or other third party material in this article are included in the article's Creative Commons license, unless indicated otherwise in a credit line to the material. If material is not included in the article's Creative Commons license and your intended use is not permitted by statutory regulation or exceeds the permitted use, you will need to obtain permission directly from the copyright holder. To view a copy of this license, visit http://creativecommons.org/ licenses/by/4.0/.

(C) The Author(s) 2020 